\title{
RANSAC algorithm for instantaneous frequency estimation and reconstruction of frequency-modulated undersampled signals
}

\author{
Igor Djurović(i)
}

Correspondence: igordj@ucg.ac.me Electrical Engineering Department, University of Montenegro, Cetinjski put 2, 81000 Podgorica,

Montenegro

\begin{abstract}
Frequency modulated (FM) signals sampled below the Nyquist rate or with missing samples (nowadays part of wider compressive sensing (CS) framework) are considered. Recently proposed matching pursuit and greedy techniques are inefficient for signals with several phase parameters since they require a search over multidimensional space. An alternative is proposed here based on the random samples consensus algorithm (RANSAC) applied to the instantaneous frequency (IF) estimates obtained from the time-frequency (TF) representation of recordings (undersampled or signal with missing samples). The O'Shea refinement strategy is employed to refine results. The proposed technique is tested against third- and fifth-order polynomial phase signals (PPS) and also for signals corrupted by noise.
\end{abstract}

Keywords: Compressive sensing, FM signals, Time-frequency analysis, Instantaneous frequency, RANSAC, Refinement

\section{Introduction}

There is growing interest in processing undersampled signals, signals with missing samples, randomly or nonuniformly sampled signals with an overall sampling rate far below the Nyquist rate. We use the term undersampled in the sequel for brevity reasons covering all mentioned scenarios. For treating such signals, we are witnessing a powerful novel paradigm called compressive sensing (CS) [1,2]. In the time-frequency (TF) signal analysis, it shed new light since TF representations are designed to be sparse, i.e., to be concentrated in the TF plane as much as possible with a large portion of the TF plane without information on a signal of interest [3-9]. Similar holds for parametric estimation where signals can be described with a significantly smaller number of parameters than the number of samples required by sampling theorem [10, 11]. However, numerous parametric estimators for polynomial phase signals (PPS) and in general for frequency modulated (FM) signals are developed in the frequency domain using classical tools such are Fourier transform (FT) or its extensions. Therefore, these techniques can be applied only to signals sampled according to the Nyquist criterion.

(c) The Author(s). 2021 Open Access This article is licensed under a Creative Commons Attribution 4.0 International License, which permits use, sharing, adaptation, distribution and reproduction in any medium or format, as long as you give appropriate credit to the original author(s) and the source, provide a link to the Creative Commons licence, and indicate if changes were made. The images or other third party material in this article are included in the article's Creative Commons licence, unless indicated otherwise in a credit line to the material. If material is not included in the article's Creative Commons licence and your intended use is not permitted by statutory regulation or exceeds the permitted use, you will need to obtain permission directly from the copyright holder. To view a copy of this licence, visit http://creativecommons.org/licenses/by/4.0/. 
Recently several techniques emerge in the spectral analysis of undersampled FM signals [12-16]. More or less, they are based on the matching pursuit or greedy algorithms performing a search over certain parametric space. Some of these strategies exhibit excellent results but they are difficult for generalization and efficient implementation for signals with more parameters due to the requirement to search over multidimensional space [17-19]. Therefore, in this paper, we are considering a strategy called RANSAC-random samples consensus algorithm-to reduce the required search space for parameters. The RANSAC is a popular tool developed mainly for video and image analysis, object tracking, etc. It has gained popularity in parametric and nonparametric signal estimation. The QML-RANSAC algorithm is proposed for the IF and PPS parameter estimation in high-noise environments [20-22]. In [20], the RANSAC has been applied to the Wigner distribution-based IF estimates. Better results in parametric estimation are achieved when the RANSAC is applied to the STFT and QML in [21]. The QML-RANSAC reduces the SNR threshold for 1-3dB to the QML results for highnoise environments. Finally, the QML is used for multicomponent signals in [22].

However, the framework considered in this research is different. Here, we are dealing with undersampled signals and all the crucial QML and QML-RANSAC steps require modification. The RANSAC algorithm is applied to IF estimates calculated from the TF representations of undersampled signals. TF representations are evaluated within the robust TF analysis framework [23]. The IF estimates are obtained maximizing the TF representation [24]. Then, multiple trials with a random sampling of the IF are generated, and for each trial, a reconstructed signal (or IF) is obtained. For precise signal reconstruction, the O'Shea refinement strategy has been adopted [25]. Based on the appropriate criterion (maximum likelihood - ML function), the best trial is selected as the algorithm output. The number of trials in the RANSAC procedure can be substantial. It means that if we have a parametric model of signal with a small number of parameters (for example quadratic phase signal or other signals with one or two parameters), it can be more efficiently processed with matching pursuit or greedy algorithm strategies. However, for signals with more parameters, for example, PPS of order three or higher, it would be better to employ the RANSAC algorithm. As will be demonstrated, an increase in the number of trials in the RANSAC algorithm is not as drastic as in the case of matching pursuit and related strategies. In general, the RANSAC algorithm complexity is not so dependent on the number of signal parameters and it represents an excellent alternative to existing strategies in the field. The proposed strategy advantage over matching pursuit techniques concerning the number of trials is analyzed and demonstrated for higher-order PPSs.

In Section 2, we have reviewed basic information about TF representation evaluation for undersampled signals followed by an introduction of the RANSAC algorithm in Section 3. The main design factor of the RANSAC-based technique is the required number of trials. It is discussed in Section 4. The simulation study is presented in Section 5. Extension for the multicomponent signal is done in Section 6 with concluding remarks and directions for further research given in Section 7.

\section{Signal model and TF representation}

Assume that an FM signal $x(n)$ is sampled according to the Nyquist criterion in equally spaced instants with total $N$ samples 


$$
x(n)=A \exp (j \phi(n)),
$$

where $A$ is signal amplitude, $\phi(n)$ is signal phase while particularly important signal feature, and the instantaneous frequency (IF) is defined as the phase derivative:

$$
\omega(n)=\phi^{\prime}(n) \text {. }
$$

In our research, the phase is modeled as a polynomial function (PPS-polynomial phase signal):

$$
\phi(n)=a_{0}+\sum_{m=1}^{M} a_{m} \frac{n^{m}}{m}
$$

where $M$ is polynomial order. The IF of this signal is:

$$
\omega(n)=\sum_{m=1}^{M} a_{m} n^{m-1}
$$

The simplest TF representation is the short-time Fourier transform (STFT) [3] reemerging recently in parametric estimation mainly due to robustness to the noise influence that cannot be achieved with nonlinear TF representations [11, 26-28]. The STFT can be written as

$$
\operatorname{STFT}_{h}(n, \omega)=\mathrm{T}\left\{x(n+k) w_{h}(k) \exp (-j \omega k) \mid k \in[-N / 2, N / 2)\right\},
$$

where $\mathrm{T}\{\}$ is an operator applied to modulated signal samples $x(n+k) w_{h}(k) \exp (-j \omega n)$. Window width is denoted as $h, w_{h}(k) \neq 0$ for $k \in[-h / 2, h / 2)$, and $w_{h}(k)=0$ elsewhere. For simplicity reasons and due to noise rejection capabilities, we use rectangular windows. In the standard STFT, operator $\mathrm{T}\{\}$ is the sum or mean. However, in the case of signals corrupted by an impulsive noise, the operator should alleviate the impulsive noise impact [23]. An overview of possible operators could be found in [23, 29, 30]. The marginal-median and L-filter forms are the most popular robust DFT/STFT variants due to simplicity and good results achieved for various impulsive, heavy-tailed, mixed Gaussian, and impulsive noise environments with operators defined respectively as:

$$
\begin{aligned}
\mathrm{T}\{y(k) \mid k \in[-N / 2, N / 2)\} & =\operatorname{median}\{\operatorname{real}\{y(k)\} \mid k \in[-N / 2, N / 2)\} \\
+j \operatorname{median}\{\operatorname{imag}\{y(k)\} \mid k \in[-N / 2, N / 2)\} & \\
\mathrm{T}\{y(k) \mid k \in[-N / 2, N / 2)\} & =\sum_{i=0}^{N-1} \alpha_{i}\left[\mathbf{r}_{i}+j \mathbf{i}_{i}\right]
\end{aligned}
$$

where $\mathbf{r}_{i}, \mathbf{i}_{i}$ are sorted elements $\mathbf{r}_{i} \leq \mathbf{r}_{i+1}, \mathbf{i}_{i} \leq \mathbf{i}_{i+1}$ from sets:

$$
\begin{aligned}
& \mathbf{r}_{i} \in\{\operatorname{real}\{y(k)\} \mid k \in[-N / 2, N / 2)\} \\
& \mathbf{i}_{i} \in\{\operatorname{imag}\{y(k)\} \mid k \in[-N / 2, N / 2)\} .
\end{aligned}
$$

Discussion about weights selection can be found in [23], but the $\alpha$-trimmed mean variant is the most common choice

$$
\alpha_{i}=\left\{\begin{array}{cc}
1 / \alpha N & i \in[N(1-\alpha) / 2, N(1+\alpha) / 2) \\
0 & \text { elsewhere }
\end{array}\right.
$$

where $\alpha$ is the trimming parameter. For $\alpha=1 / N$, it gives the marginal-median filter STFT, while for $\alpha=1$, it produces the standard STFT. 
The IF as a crucial feature of the FM signals can be estimated maximizing STFT:

$$
\omega_{h}(n)=\arg \max _{\omega}\left|\operatorname{STFT}_{h}(n, \omega)\right| .
$$

This IF estimator is biased with the bias increasing as window width does. However, the estimator variance decreases with increasing of the window width, meaning there is a trade-off in the window width selection. Determination of the optimal window width is a difficult task since bias is signal-dependent. Strategies for achieving the trade-off are reviewed in [24]. However, almost all of them are developed for favorable conditions: signal recorded according to the sampling theorem and moderate amount of noise. Parametric estimators face harsh conditions, i.e., signal-to-noise ratio (SNR) even below $\mathrm{OdB}$ and in the considered case dealing with undersampled signals.

The bias was the reason preventing usage of the STFT-based techniques in the parametric estimation for a long time. A recently proposed technique called the quasi maximum likelihood (QML) estimates parameters of the PPS by the polynomial regression of the IF estimate [11, 26-28]. Residual errors caused by the bias and variance of the IF estimator are reduced with the O'Shea refinement technique [25]. The final estimate is selected by simple criterion function, i.e., matching correlator or ML penalty function, giving robust technique to determine optimal window width in the STFT and final algorithm output.

The main challenge in the considered research is that the Nyquist criterion is not satisfied for the considered undersampled signals. Samples are here randomly or nonuniformly distributed or with a large percentage of missing recordings. In the robust spectral and TF analysis framework, these missing samples can be treated as outliers with positions of outliers known in advance. However, an additional challenge of the CS framework is the fact that the percentage of available to samples required by the Nyquist criterion can be extremely low (even $5-10 \%$ ) while the percentage of impulses considered within robust spectral analysis rarely surpassed $50 \%$ and often it is below $25 \%$.

Denote instants with available samples as $n_{i}, i=1, \ldots, K$ where $K \ll N$. For simplicity, reasons assume ordered samples $n_{i} \leq n_{i+1}$. Our goal is a precise estimation of phase parameters of signal $\left\{a_{0}, a_{1}, \ldots, a_{M}\right\}$ and consequently signal reconstruction based on available signal samples $x\left(n_{i}\right), i=1, \ldots, K$.

In the next section, we present the RANSAC-based procedure for the estimation of the parameters of undersampled PPSs.

\section{RANSAC for signal reconstruction}

First of all, it is important to discuss how to evaluate the TF representation for undersampled signals. For considered instant $n$, we are looking for available samples from set $\mathbf{Q}=\left\{n_{i}, i=1, \ldots, K\right\}$ that are within the window:

$$
n_{i} \mid n_{i} \in[n-h / 2, n+h / 2)
$$

Then, the STFT output can be calculated as:

$$
\operatorname{STFT}_{h}(n, \omega)=\mathrm{T}\left\{x\left(n_{i}\right) \exp \left(-j \omega\left(n_{i}-n\right)\right) \mid n_{i} \in[n-h / 2, n+h / 2)\right\} \quad h \in \mathbf{H} .
$$

Operator $\mathrm{T}\{\}$ is selected here to average available modulated samples within the considered window. It can be treated as the L-filter STFT version. The STFTs are evaluated for various window lengths due to the different behavior of the corresponding IF 
estimators related to bias and variance for the applied window width. We intend to have a set of STFTs with diverse elements (wide windows - robust to the noise influence but can experience emphatic bias, narrow windows - small bias, and emphatic noise influence and all cases between) to be able to achieve reachable performance under various circumstances.

The IF estimation is performed by maximizing STFT (3). For a small ratio available to samples required by Nyquist criterion $(K / N)$ and narrow windows, there can be no available samples in the neighborhood $n_{i} \in[n-h / 2, n+h / 2)$. It is adopted that the IF estimate is zero in this region but in practice, it could be applied an interpolation using available IF estimates to further improve results. Several IF samples are selected in each trial of the RANSAC. The IF (or signal) is reconstructed using these samples. However, due to several reasons (missing samples, outliers in the IF estimate, etc.), the reconstructed IF (or signal) can be inaccurate. Therefore, the random samples selection should be repeated multiple times, and the most accurate among reconstructed signals/ IF estimates is selected based on an appropriate criterion. A window width from set $\mathbf{H}$ and IF estimate samples $\hat{\omega}_{h}(n)$ are randomly selected in each trial of the proposed RANSAC procedure. Polynomial parameters of the signal phase are obtained using polynomial regression/interpolation. The STFT-based IF estimation of higher-order PPSs exhibits bias even for signals sampled according to the Nyquist criterion [26]. Then, the O'Shea refinement is applied to improve the accuracy of the PPS coefficients estimation toward the Cramer-Rao lower bound. In the case of undersampled signals, refinement is even more important since it can improve relatively inaccurate estimates without outliers what cannot be done for IF estimate samples with outliers.

Now, we can summarize the proposed RANSAC algorithm in the subsequent subsections. Firstly, a pseudo-code of the proposed technique is given followed by a detailed explanation of the crucial algorithm steps.

\subsection{Pseudocode}

\subsubsection{RANSAC algorithm with the O'Shea refinement for parameter estimation of} undersampled signals

Input: PPS signal $x\left(n_{i}\right) i=1, \ldots, K$ of order $M$ where samples are given in increasing order $n_{i} \leq n_{i+1}$.

Step I Evaluation of the STFTs with various window widths and obtaining corresponding IF estimates.

For $h \in \mathbf{H}$

$$
\begin{aligned}
& \operatorname{STFT}_{h}(n, \omega)=\mathrm{T}\left\{x\left(n_{i}\right) \exp \left(-j \omega\left(n_{i}-n\right)\right) \mid n_{i} \in[n-h / 2, n+h / 2)\right\} \quad h \in \mathbf{H} \\
& \hat{\omega}_{h}(n)=\arg \max _{\omega}\left|\operatorname{STFT}_{h}(n, \omega)\right| .
\end{aligned}
$$

$\underline{\text { EndFor }}$

Step II Initialization of criterion functions:

$$
J_{\max }=0 \quad J_{\max }^{\prime}=0 .
$$

Step III Trials with random window length and random IF samples ( $\Lambda$ trials in total).

$\underline{\text { For }} \lambda=1: \Lambda$

Step III.A Random selection 
Randomly select $h_{\eta} \in \mathbf{H}$ and IF samples $\hat{\omega}_{\eta}\left(n^{j}\right) \quad \eta \in \mathbf{H} \quad n^{j} \in \mathbf{Q}, n^{j}<n^{j+1}, j \in[1, M]$.

Step III.B Rough parameter estimation

Perform polynomial regression/interpolation of the IF estimation samples:

$$
\left\{\hat{a}_{1}^{\lambda}, \ldots, \hat{a}_{M}^{\lambda}\right\}=\left(\boldsymbol{\Gamma}^{T} \boldsymbol{\Gamma}\right)^{-1} \boldsymbol{\Gamma}^{T}\left[\hat{\omega}_{\eta}\left(n^{1}\right) \hat{\omega}_{\eta}\left(n^{2}\right) \cdots \hat{\omega}_{\eta}\left(n^{M}\right)\right]^{T}
$$

where $\boldsymbol{\Gamma}$ is $M \times M$ matrix with elements $\gamma_{i, j}=j\left(n^{i}\right)^{j-1}$ and $i, j \in[1, M]$. This is a rough estimate in the QML-algorithm terminology.

Step III.C O'Shea refinement

Dechirping with parameters available from the rough stage:

$$
x^{\prime}\left(n_{i}\right)=x\left(n_{i}\right) \exp \left(-j \sum_{m=1}^{M} \frac{\hat{a}_{m}^{\lambda}\left(n_{i}\right)^{m}}{m}\right) .
$$

Phase unwrapping:

$$
\hat{\phi}\left(n_{i}\right)=\operatorname{unwrap}\left\{\operatorname{phase}\left\{x^{\prime}\left(n_{i}\right)\right\}\right\} .
$$

Polynomial regression of the signal phase:

$$
\left\{\delta \hat{a}_{0}^{\lambda}, \delta \hat{a}_{1}^{\lambda}, \ldots, \delta \hat{a}_{M}^{\lambda}\right\}=\left(\boldsymbol{\Xi}^{T} \boldsymbol{\Xi}\right)^{-1} \boldsymbol{\Xi}\left[\hat{\boldsymbol{\phi}}\left(n_{1}\right) \hat{\boldsymbol{\phi}}\left(n_{2}\right) \cdots \hat{\boldsymbol{\phi}}\left(n_{K}\right)\right]^{T},
$$

where matrix $\Xi$ has $(M+1) \times K$ elements given as $\xi_{i, j}=n_{i}^{j}, \quad i \in[1, K], j \in[0, M]$.

Step III.D Fine estimate is obtained as:

$$
\begin{aligned}
& \hat{a}_{m}^{\prime \lambda}=\hat{a}_{m}^{\lambda}+\delta \hat{a}_{m}^{\lambda} \quad m=1,2, \ldots, M \\
& \hat{a}_{0}^{\prime \lambda}=\delta \hat{a}_{0}^{\lambda} .
\end{aligned}
$$

Step III.E Evaluation of criterion function (for estimates with and without refinement) [31, 32]:

$$
\begin{aligned}
& J(\lambda)=\left|\sum_{i=1}^{N_{c}} x\left(n_{i}\right) \exp \left(-j \sum_{m=1}^{M} \frac{\hat{a}_{m}^{\lambda}\left(n_{i}\right)^{m}}{m}\right)\right| \\
& J^{\prime}(\lambda)=\left|\sum_{i=1}^{N_{c}} x\left(n_{i}\right) \exp \left(-j \sum_{m=1}^{M} \frac{\hat{a}_{m}^{\prime \lambda}\left(n_{i}\right)^{m}}{m}\right)\right| .
\end{aligned}
$$

If $J(\lambda)>J$, set $J=J(\lambda)$, and update estimates as $\left\{\hat{a}_{1}, \ldots, \hat{a}_{M}\right\}=\left\{\hat{a}_{1}^{\lambda}, \ldots, \hat{a}_{M}^{\lambda}\right\}$. EndIf

If $J(\lambda)>J^{\prime}$ set $J=J(\lambda)$ and update $\left\{\hat{a}_{0}^{\prime}, \hat{a}_{1}^{\prime}, \ldots, \hat{a}_{M}^{\prime}\right\}=\left\{\hat{a}_{0}^{\prime \prime}, \hat{a}_{1}^{\prime \lambda}, \ldots, \hat{a}_{M}^{\prime \lambda}\right\} . \underline{\text { EndIf. }}$.

\section{EndFor}

Output: The estimated set of phase coefficients $\left\{\hat{a}_{0}^{\prime}, \hat{a}_{1}^{\prime}, \ldots, \hat{a}_{M}^{\prime}\right\}$ or $\left\{\hat{a}_{1}, \ldots, \hat{a}_{M}\right\}$ can be used to reconstruct signal $x(n)=\hat{A} \exp \left(-j \sum_{m=1}^{M} \frac{\hat{a}_{m} n^{m}}{m}\right)$ where the amplitude is estimated as

$$
\hat{A}=\operatorname{mean}\left\{\left|x\left(n_{i}\right) \exp \left(-j \sum_{m=1}^{M} \frac{\hat{a}_{m}\left(n_{i}\right)^{m}}{m}\right)\right|\right\} .
$$




\subsection{Comments on the algorithm}

Due to the large percentage of missing samples in recordings, it can be expected a significant percentage of outliers in the IF estimate. Therefore, the RANSAC procedure is employed to select, among numerous wrong estimates, accurate IF estimate samples. Parameters of the signal phase are estimated allowing accurate signal reconstruction $[26,27]$. The STFT is used in the QML-RANSAC algorithm for the IF estimation in the rough algorithm stage. Here, due to missing samples evaluation is performed with the modified (robust) STFT form in Step I.

Two criterion functions are initialized in Step II. The first is evaluated without the O'Shea refinement and used only for reference to demonstrate the refinement stage impact on the algorithm accuracy. Both criterion functions evaluated in Step III.E correspond to the ML optimization. Polynomial regression is applied in rough (4) and fine (6) estimation stages. However, it should be noted that in the rough stage it is performed only on $M$ randomly selected samples of the IF estimate, while in the fine stage it is performed on all available $K$ signal phase samples. Weight coefficients in matrices $\Gamma$ and $\boldsymbol{\Xi}$ are different since evaluations in (4) and (6) are performed for different functions (IF and phase).

There are also some issues in the O'Shea refinement algorithm worth to be discussed. They are related to two important steps in the refinement procedure: filtering and phase unwrapping. The signal filtering is commonly performed in the refinement stage of the algorithm in both the QML and QML-RANSAC to reduce noise-like effects from the estimate and from this point of view it could be useful:

$$
\widehat{x^{\prime}}\left(n_{i}\right)=\frac{1}{2 l+1} \sum_{k=-l}^{l} x^{\prime}\left(n_{i+k}\right) .
$$

However, it can introduce additional errors distorting dechirped signal $x^{\prime}\left(n_{i}\right)$ used in the further procedure. These errors can be emphatic when neighbor signals are far from each other. In our experiments, filtering is not applied (set $l=0$ in (7)) but it remains for further research on how it can be utilized especially for signals corrupted by a significant amount of additive noise. A similar problem holds with the phase unwrapping since it can be inaccurate for a low number of available samples (low $K / N$ ratio). However, the phase unwrapping is applied in our experiments as in $[26,28]$ with good results but some enhancement of this procedure is probably possible remaining for further research.

\section{Number of trials in the RANSAC algorithm}

There are two design parameters in the algorithm: the set of window lengths $\mathbf{H}$ and the required number of trials $\Lambda$. Recommendation from [31] is adopted for window lengths set $\mathbf{H}$ : up to 10 windows with widths growing according to the geometrical progression from narrow (8 samples) toward the wide window (256 samples). Therefore, the issue of selecting the number of trials $\Lambda$ is discussed in this section. This is a crucial setup parameter of the RANSAC algorithms, and it will be demonstrated that it is significantly smaller than a search space in matching pursuit techniques for cubic-phase and PPSs of a higher order. The required number of trials for the RANSAC algorithm executed in time, spatial, or feature domains is a well-researched topic [33]. However, it is 
still of interest for the indirect estimation of parameters from phase or a signal IF. Note that issue of how outliers (or missing samples) influence IF estimates is not well studied in the robust TF framework even for signals sampled according to the Nyquist criterion. There are some derivations but they are almost always related to asymptotic conditions or specific noise environments with the notable exception [17]. A simple experiment is employed here to study how missing samples influence the appearance of outliers in the IF estimates.

Experiment: For simplicity a linear FM signal:

$x(n)=\exp \left(j \pi n^{2} / 2^{11}\right)$ with $n \in\left[-2^{9}, 2^{9}\right]$ is considered with the IF

$$
\omega(n)=\pi n / 2^{10} n \in\left[-2^{9}, 2^{9}\right] .
$$

The STFT is evaluated with various window widths $h$ and number of available samples $K$ (or ratio $K / N$ ) in the considered domain of $\left[-2^{9}, 2^{9}\right]$ (in each trial $K$ samples are randomly selected from $N=2^{10}+1=1025$ samples). Our goal is to measure the number of IF estimation outliers in the spectral domain for different $K / N$ and $h$. The IF estimates far from the true values:

$$
\left|\omega_{h}(n)-\omega(n)\right|>\Delta \omega
$$

are classified as outliers. The threshold is set to $\Delta \omega=0.08 \pi$. Results (percentage of correct IF estimated within $\Delta \omega$ for various $h$ and $K / N$ ) obtained with 100 trials are depicted in Fig. 1. The main finding from the experiment is that the percentage of outliers in IF estimates is smaller than in the time domain, i.e., the percentage of correctly estimated IF samples is larger than $K / N$ ratio in almost all cases of interest. The procedure to calculate the STFT does not increase the percentage of outliers in the IF estimate comparing to the percentage of missing samples. Then, the RANSAC algorithm can be applied to this feature with at least the same performance as in the case of direct application of the RANSAC algorithm to polynomial lines. Some loss of performance is

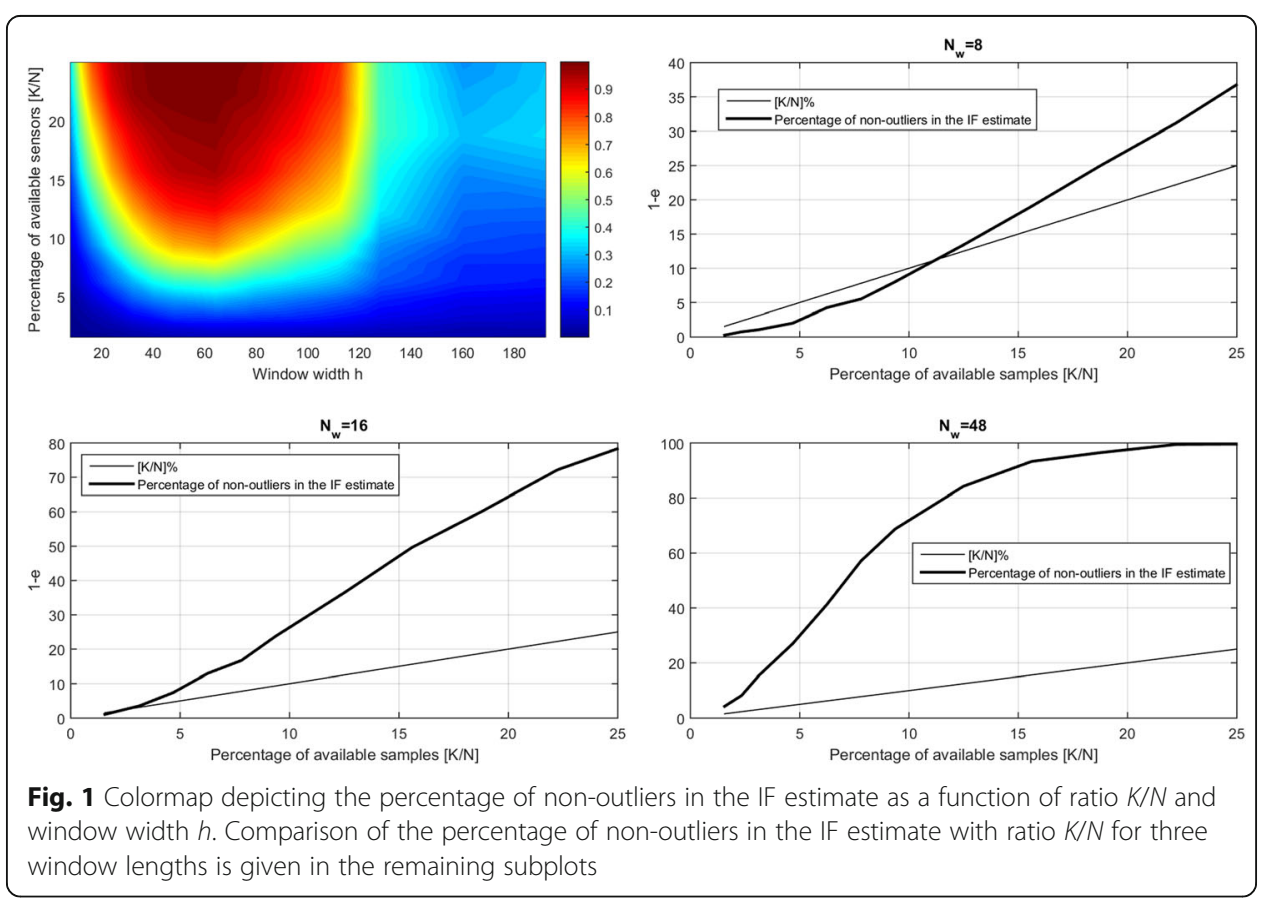


expected in the case of the PPS of higher-order due to bias in the STFT-based IF estimates but again, as it will be demonstrated within numerical examples, the developed RANSAC-based procedure achieves satisfactory accuracy.

Figure 1, northwest, depicts the percentage of correct IF estimates vs. the ratio of available and samples required by the Nyquist criterion $K / N(y$-axis) and window width $h$ in the STFT ( $x$-axis). The corresponding colorbar is also given on the right-hand side showing clearly that only for extremely narrow windows (with less than 20 samples), the number of non-outliers cannot be above 50\%. The hot (red, orange, and yellow) area in this subplot depicts that it is possible to achieve less than $30 \%$ of outliers in the IF estimate with window widths between $h=20$ and $h=120$ samples even for $K / N=10$ $15 \%$. It is presented more clearly in the remaining subplots of Fig. 1 with this statistic given for three typical window lengths ( $h=8$ (very narrow window), $h=16$, and $h=48$ samples). The thin line represents ratio $K / N$ (the same as the $x$-axis) while the thick line is the percentage of correct IF estimate within limits (8) around exact IF. It can be seen that only for extremely narrow window $h=8$ and/or for small ratio $K / N$ bellow $K /$ $N=10 \%$, percentage of outliers in the IF estimate is larger than the number of missing samples in the original signal. This simple experiment shows that it is safe to use IF estimate for the RANSAC algorithm application with at least the same performance as in the case of polynomial functions fitting in the feature space. Note that the IF is a derivative of the signal phase with a decremented number of parameters concerning phase and the RANSAC application is further simplified in this domain requiring a smaller number of trials. Constant phase parameter $a_{0}$ and amplitude $A$ can be estimated by the trivial procedure when other phase parameters are known.

For further results clarification and showing something that is hidden behind given statistics, we have shown STFTs for a single trial for $K / N=7.8 \%$ (upper row) and $K / N=$ 18.7\% (bottom row) in Fig. 2. Columns represent various window widths: $h=16, h=48$,

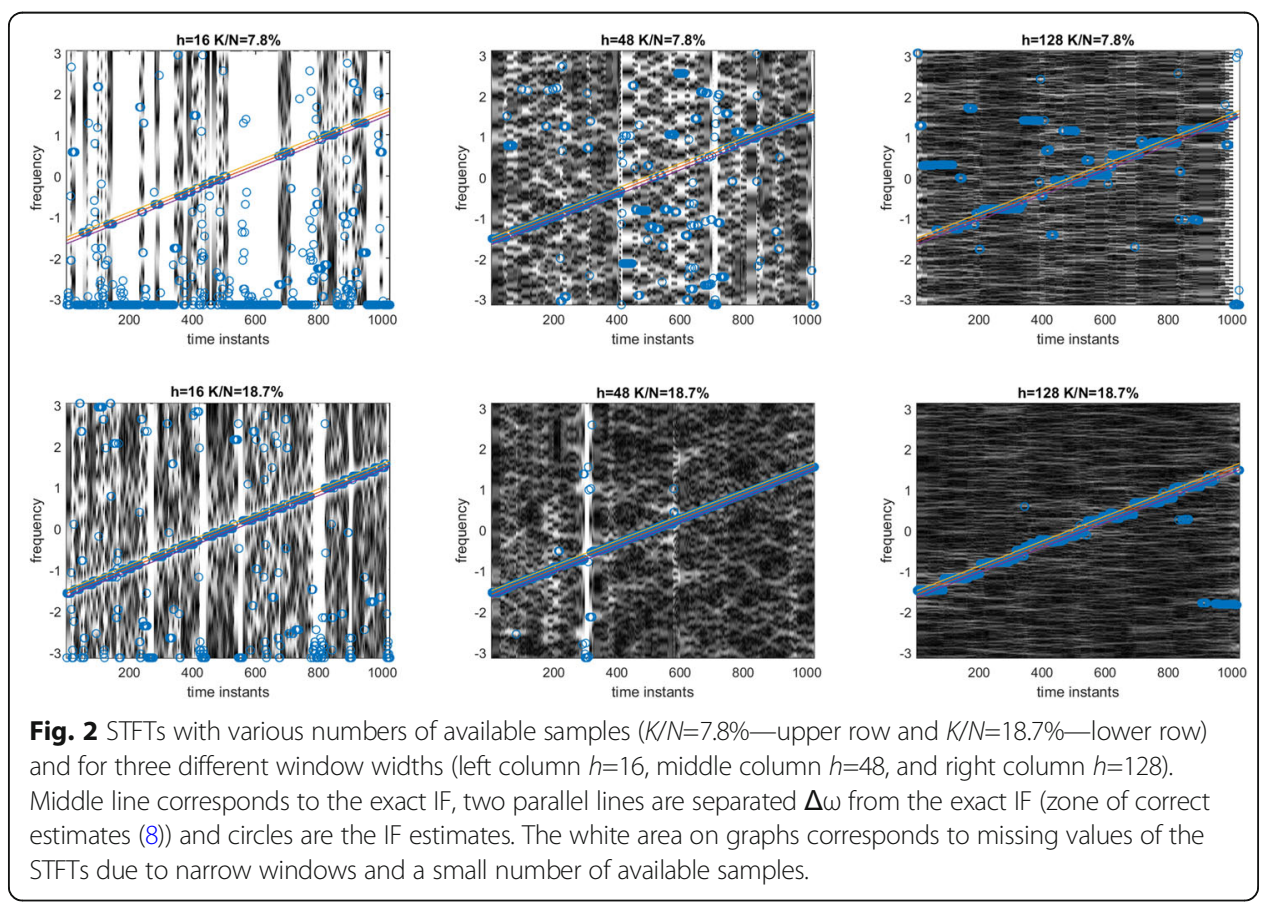


and $h=128$ samples, respectively. Straight-line in subplots is true IF with two parallel lines representing a range of $\Delta \omega$ around exact IF. The IF estimates are depicted with circles. Large white space in STFTs in the first subplot corresponds to a zone without a single available sample (narrow window and a small number of available samples). Percentages of IF estimates that are not outliers, i.e., within range $\Delta \omega$, as a function of ratio $K / N$ and window width for considered trials are:

$P(N / K=7.8 \%, h=16)=20.5 \% \quad P(N / K=7.8 \%, h=48)=57.1 \% \quad P(N / K=7.8 \%, h=128)=24.5 \%$

A drop of performance for a wide window of $h=128$ is evident which is also clearly observed from Fig. 1 (large blue array of a low percentage of correct IF estimates). However, it is partially caused by adopted statistics and range of correct IF estimates $\omega(n) \pm \Delta \omega$ what is visible from Fig. 2 , southwest. Therefore, it is probably not correct to tell that there is a large number of outliers in the IF estimate for wide windows since the significant percent of estimates is close to the non-outliers range. Such behavior is known in the case of wide rectangular windows in the FT and STFT (Gibbs phenomenon and related effects). Note that smooth windows are not used in the STFT since they tend to increase the number of outliers so the rectangular window in the STFT is adopted even with the described phenomena.

The RANSAC algorithms are usually designed in such a manner to produce correct estimation with a probability that is above a selected threshold [32]. Commonly, this probability is set above $p>0.99$. Assume that percentage of outliers in the IF estimate is $e$. For polynomial interpolation, we need that all $M$ randomly selected IF samples correspond to the signal IF. Then, the number of trials $\Lambda$ in the RANSAC procedure should satisfy [32]:

$$
\left(1-(1-e)^{M}\right)^{\Lambda}=1-p
$$

After simple derivations it follows:

$$
\Lambda=\log (1-p) / \log \left(\left(1-(1-e)^{M}\right) . .\right.
$$

For example, for $p=0.99, e=0.5$, and $M=5$, it is required $\Lambda=146$ trials in the RANSAC to achieve the desired accuracy. However, more interesting cases are with a higher percentage $e$ of outliers in the IF estimation. The number of outliers can be increased due to a smaller number of available samples or for higher-order PPSs due to the signal spreading in the TF domain. For $e=0.8$, required number of trials increases to $\Lambda=14390$ while for $e=0.9$, it is going up $\Lambda=46000$. Table 1 summarizes results related to the

Table 1 Required number of trials $\wedge$ to achieve a probability of success in the RANSAC procedure of $p=0.99$ as a function of the percentage of outliers $e$ in the IF and order of polynomial $M$

\begin{tabular}{lllll}
\hline $\boldsymbol{\Lambda}$ & $\boldsymbol{e}=\mathbf{0 . 3}$ & $\boldsymbol{e}=\mathbf{0 . 5}$ & $\boldsymbol{e}=\mathbf{0 . 8}$ & $\boldsymbol{e = 0 . 9}$ \\
\hline$M=3$ & 7 & 35 & 574 & 4603 \\
$M=5$ & 12 & 146 & 14389 & 460515 \\
$M=6$ & 20 & 588 & 359777 & 46051700 \\
$M=10$ & 41 & 4714 & 44972363 & 46051698048 \\
\hline
\end{tabular}


number of trials in the case of various orders of polynomial $M$ and the percentage of outliers $e$ in the IF estimate. Now, it is possible to assess the benefits of the application of the RANSAC procedure to direct search techniques employed in the matching pursuit and greedy strategies.

Assume that for the direct search techniques the smallest number of parameters required for search along a single dimension is of order $N_{S}=100$. Then, the required search space for estimation of parameters of a signal of order $M$ is:

$$
100^{M-1}=10^{2 M-2}
$$

Note that in the matching pursuit strategies, it is required to search over (M-1)dimensional parametric space since $a_{1}$ parameter can be estimated using (robust) FT forms after estimating higher-order parameters.

From Table 1, it follows that for $M=3$, it is better to employ the RANSAC for $e \leq 0.9$ since direct search requires at least $10^{4}=10000$ trials. For $e=0.9227$, the required number of trials in the RANSAC procedure is equal to a search space of $100 \times 100$ different parameters $a_{2}$ and $a_{3}$ (this is not enough in numerous applications and often additional iterative procedures are applied for results refinement). In the case of $M=5$, it is required $100^{4}=10^{8}$ search space in the matching pursuit techniques what is almost three orders of magnitude more than required by the RANSAC for $e=0.9$ and the RANSAC becomes less efficient only for $e>0.9659$ (extremely high percentage of outliers). Therefore, it can be concluded that the RANSAC is a useful tool to reduce the calculation complexity of the direct search in the considered case. Also, for a known percentage of IF estimate outliers (as demonstrated previously it can be approximated or at least overestimated with the number of missing samples), $\Lambda$ can be significantly reduced, i.e., the number of trials can be selected in dependence on the percentage of IF estimate outliers (or percentage of missing samples) while in the matching pursuit techniques it is constant. The RANSAC algorithm accuracy is considered in the next section with numerical examples.

\section{Results and discussion}

Now, we can proceed with numerical analysis of the proposed technique on several case studies.

Example 1.

Consider a cubic phase signal:

$$
x(n)=\exp \left(j 4 \pi\left(-n^{3} / 2048+128 n\right) / 2048\right) n \in[-512,512]
$$

with the IF:

$$
\omega(n)=4 \pi\left(-3 n^{2} / 2048+128\right) / 2048 .
$$

Figure 3 presents IF estimation for 100 independent realizations of the RANSAC algorithm for four $K / N$ values from $7.8 \%$ ( $K=80$ samples) to $15.6 \%$ ( $K=160$ samples). The right column shows results achieved without refinement while the left column gives results for the proposed approach with the O'Shea refinement. The RANSAC is applied with $10^{4}$ trials. It can be seen a relatively large number of outliers for $K / N=$ $7.8 \%$ without refinement while in the case of the O'Shea refinement percentage of outliers is moderate (quantified below). However, for $K / N=9.4 \%$ there are no outliers in 


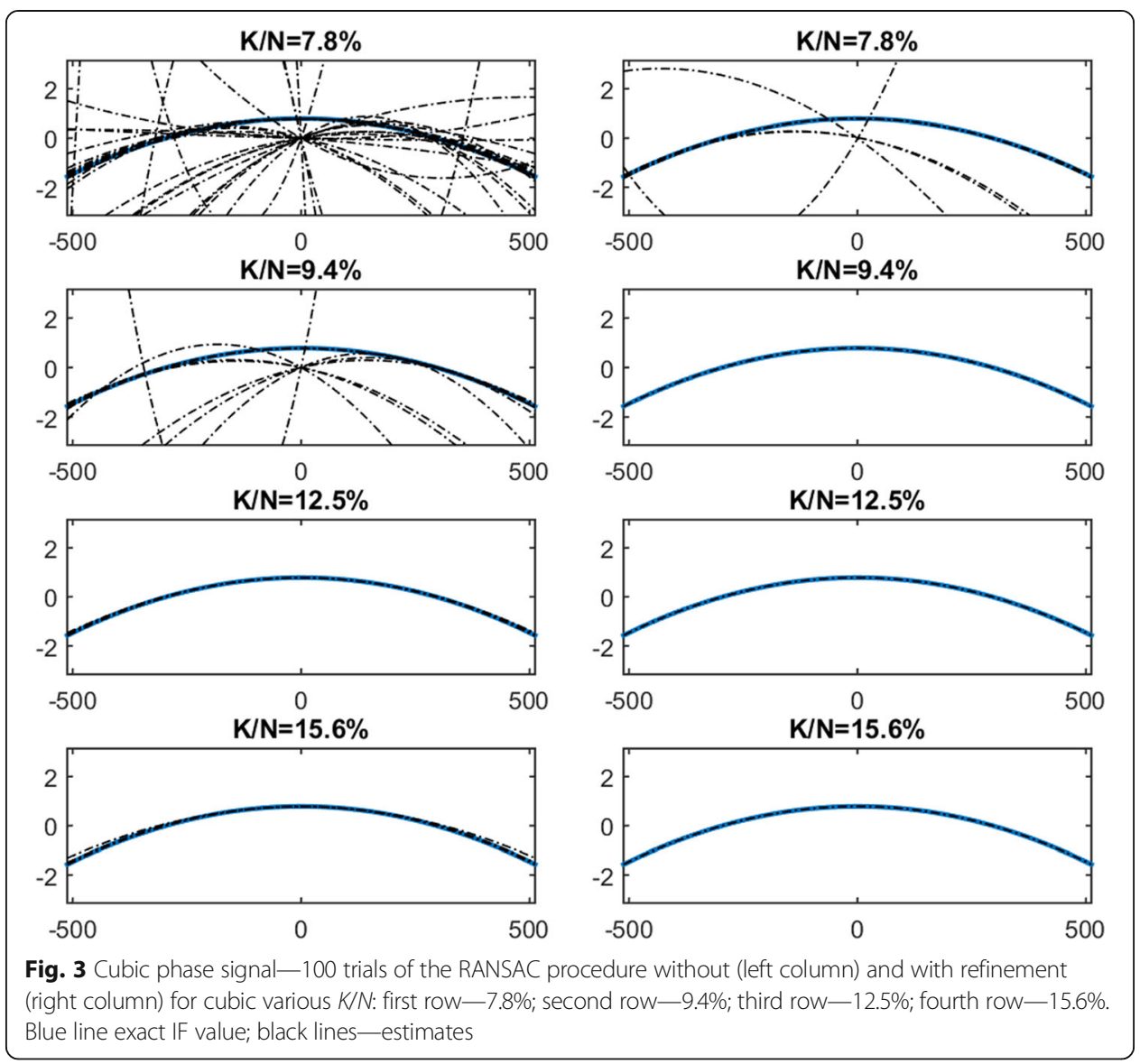

the case of the O'Shea refinement while there are still some in the case when the O'Shea refinement is not applied. For $K / N=12.5 \%$ (third row), there are no outliers in both procedures.

Besides, two measures are adopted for statistical study:

Mean absolute error - MAE

$$
10 \log _{10}\left\{\frac{1}{N_{T}} \sum_{i=1}^{N_{t}} \frac{1}{N} \sum_{n=1}^{N}|\hat{\omega}(n)-\omega(n)|\right\}[\mathrm{dB}]
$$

Mean maximal error - MME

$$
10 \log _{10}\left\{\frac{1}{N_{T}} \max \{|\hat{\omega}(n)-\omega(n)|, n \in[1, N]\}\right\}[\mathrm{dB}]
$$

where $N_{T}$ is the number of independent signal realization (selection of $K$ random samples among $N$ available). IF estimate is calculated for signal phase coefficients obtained in the proposed procedure and denoted as $\hat{\omega}(n)$. Both of these measures are calculated and depicted in Fig. 4 as a function of $K / N$. The significant improvement achieved with the O'Shea refinement application is obvious.

At about $K / N=7 \%$, both procedures achieve significant improvement while the refinement gives a huge enhancement in the logarithmic scale. It should be noted that for example for $K / N \geq 8 \%$, the MME for the proposed technique with the O'Shea refinement is almost perfect $M M E=5.4 \times 10^{-16}$, i.e., on the level close to the machine precision. 
Also, the proposed technique is tested and compared with the case when the refinement is not applied by the percentage of IF estimation outliers. We adopted that an IF estimation outlier has the MAE above $\Delta \omega=0.08 \pi$. Figure 5 depicts the percentage of outliers. It can be noted that there are no outliers in both procedures for $K / N \geq 13 \%$ when performance can be evaluated using measures like in Fig. 4. However, for $K / N<$ $13 \%$, the refinement brings significant improvement. For example, for $K / N=8 \%$, there are no outliers in the IF estimation in the case of application of the refinement but there is $7 \%$ of outliers when the refinement is not applied. For $K / N=6.6 \%$, the percentage of outliers in the case of application of the refinement is only $4 \%$ while in the case without refinement it is $21 \%$, for $K / N=5.3 \%$ the proposed technique with refinement has only $11 \%$ outliers while without refinement it has almost 4 times more, i.e., $43 \%$. It demonstrates the usefulness of the refinement procedure even in this unfavorable scenario with missing samples.

Example 2.

Example 1 considers the cubic phase (third-order PPS) signal. The QML is proposed for higher-order PPSs since it can avoid multidimensional search over parameter space. In the case of signals with missing samples, the importance of avoidance of the multidimensional search is even more important to control the complexity of the search procedure as demonstrated in the previous section. The effectiveness of the proposed technique is tested on a fifth-order PPS $(M=5)$ with the IF:

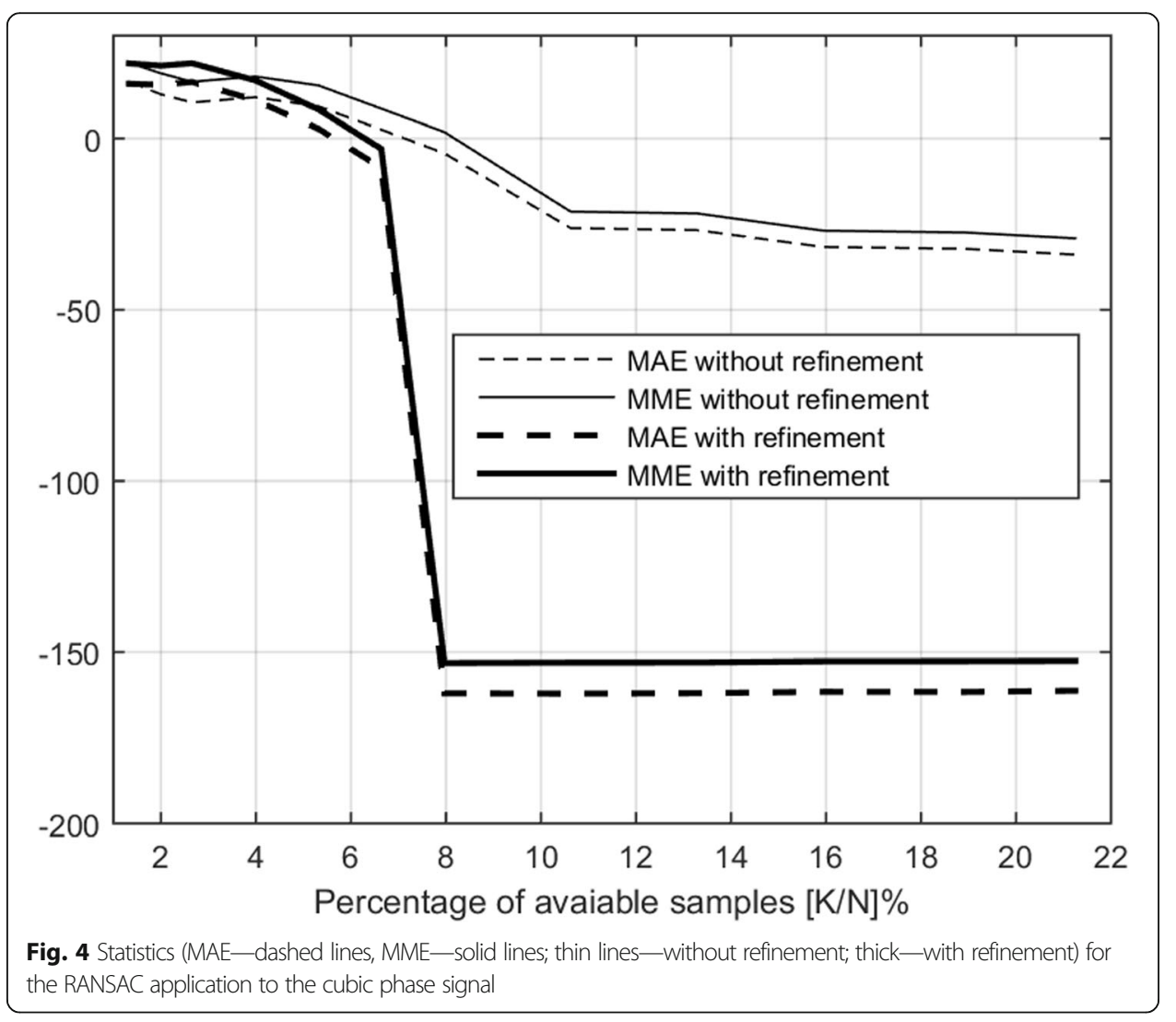




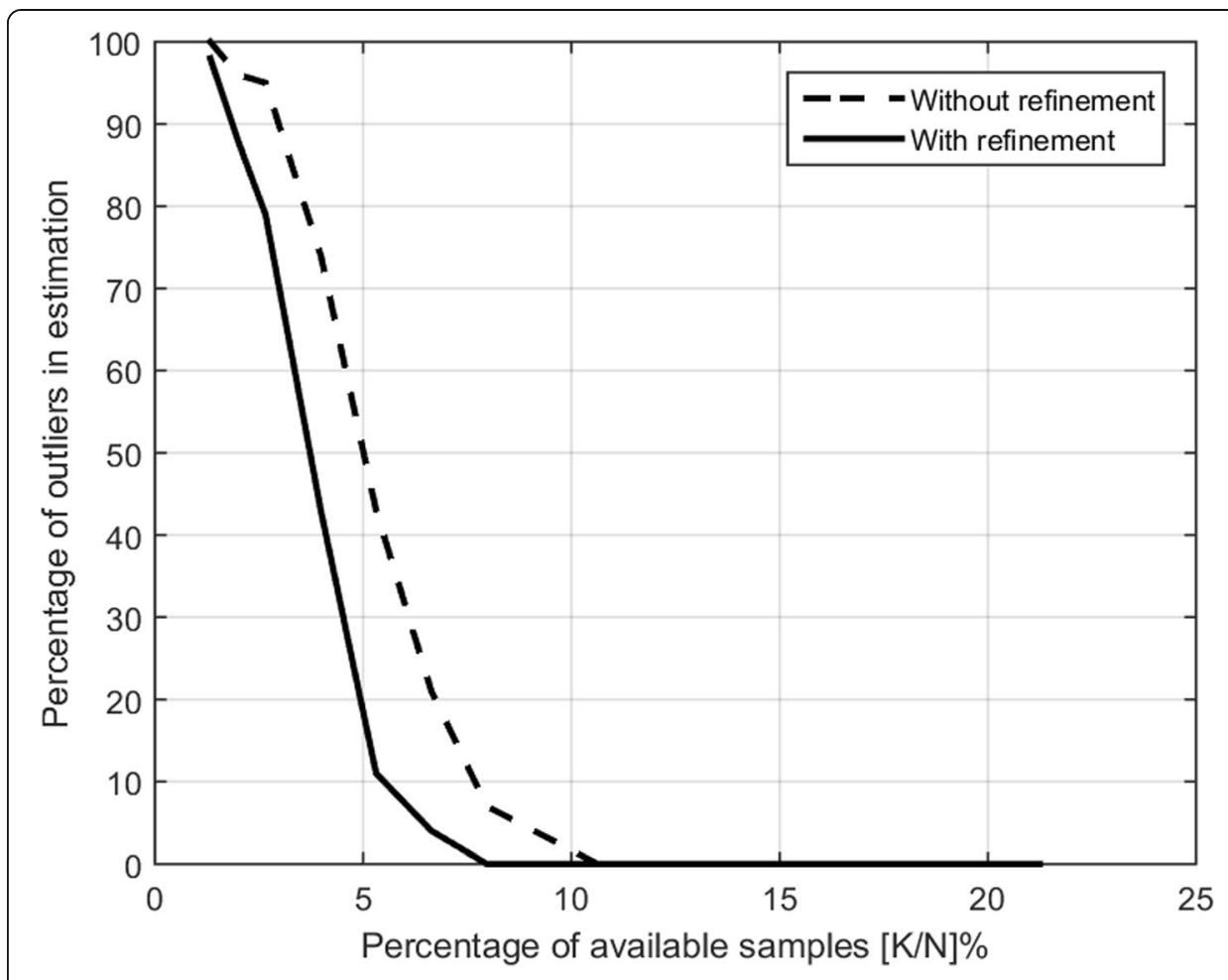

Fig. 5 Percentage of outliers in the IF estimation for the cubic phase signal: dashed line-without refinement; dotted line-with refinement

$$
\omega(n)=2 \pi\left(9.7(n / N)^{4}-2.25(n / N)^{2}-1 / 8\right) n \in[-512,512] .
$$

For the PPS with $M=5$, it is required to have more trials in both matching pursuit and the RANSAC procedures. For matching pursuit, it is conservative to tell that it is needed $N_{S}^{M-1}=100^{4}=10^{8}$ search space, where is $N_{S}$ is the size of search space for each phase parameter here with a relatively small value of $N_{S}=100$. For the RANSAC procedure, we can estimate the required number of trials (9) where $p$ is the percentage of correct estimates, and $e$ is the percentage of outliers in the IF estimate. In the RANSAC procedure, it is common to take $p=0.99$ and we are willing that our procedure works for example in the case of $e=0.9$ IF estimate outliers. Then, the required number of trials is about $\Lambda=4.6 \times 10^{5}$. This is several orders of magnitude less than in the case of matching pursuit. However, we limited $\Lambda$ to $10^{5}$ in this case. It should be recalled two effects that additionally influence the RANSAC accuracy: the first effect is positive, as already discussed within the previous section, the percentage of outliers in the STFT-based IF estimate is smaller than in the input signal; the second is negative, with an increase of the polynomial order in the signal phase the bias in the IF estimate is increased and the STFT is blurred what can introduce additional outliers in the estimate. Limiting the number of trials below the calculated value of $\Lambda=4.6 \times 10^{5}$ demonstrates that positive effects prevail.

However, as it can be seen from Fig. 6 where 100 realizations of the RANSAC procedure are shown, without (left) and with refinement (right column) obtained accuracy is good. It can be seen that for $K / N=12.5 \%$, the proposed RANSAC procedure with implemented refinement achieves almost perfect results with minor errors at interval borders. Without refinement, it is not possible to achieve estimation without 
outliers for $K / N<16 \%$, and also, it can be seen emphatic border effects caused by errors in polynomial regression (interpolation). As can be seen, this inaccuracy is almost completely removed by the refinement procedure.

The MAE and MME are given for the fifth-order PPS in Fig. 7. It can be seen similar behavior as in the case of the third-order PPS but with more samples required to achieve "perfect" accuracy than for the third-order signal what can be expected.

The percentage of outliers for $K / N=8 \%$ is $8 \%$ while for $K / N \geq 11 \%$, there are no outliers in the IF estimation (Fig. 8). However, for an algorithm without refinement, there are always some outliers due to errors in the IF estimation obtained by polynomial regression on borders of the considered interval.

We have compared obtained results with those that can be achieved by maximizing response over a dictionary of following elements $a_{2} \in[-\pi / 1024, \pi / 1024], a_{3} \in[-0.01 \pi /$ $1024,0.01 \pi / 1024], \quad a_{4} \in\left[-10^{-5} \pi / 1024,10^{-5} \pi / 1024\right]$, and $a_{5} \in\left[-10^{-8} \pi / 1024,10^{-8} \pi / 1024\right]$.

Estimation of $a_{1}$ is performed in the FT domain after dechirping signal $x(n)$ with $\exp ($ $\left.-j \sum_{m=2}^{M} \hat{a}_{m} n^{m} / m\right)$. In the first experiment, there are $N_{S}=21$ elements in each of four sets meaning that search is performed over $21^{4} \approx 1.95 \times 10^{5}$ elements what is almost twice the number of trials for the RANSAC algorithm. In the second experiment, we have

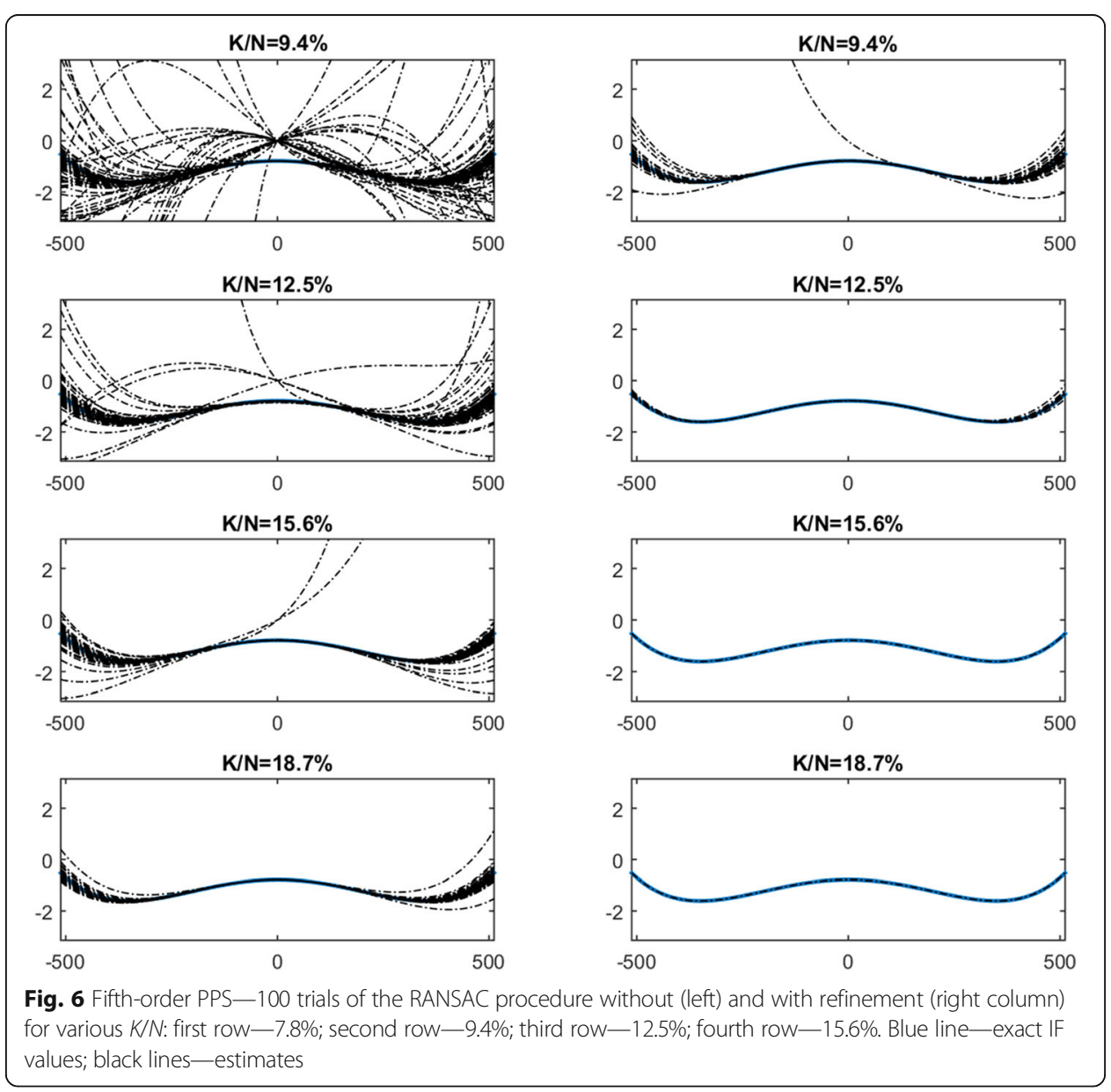




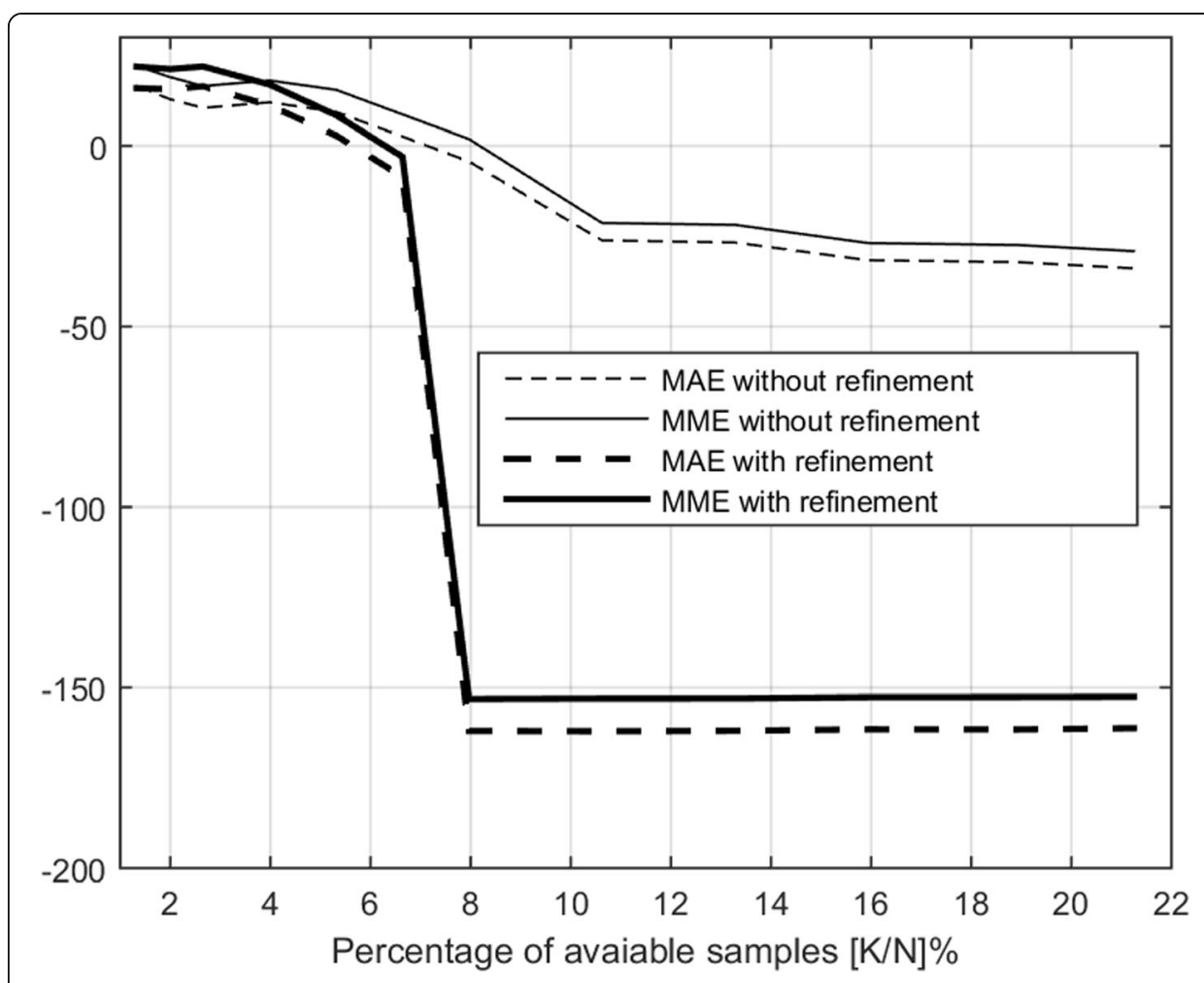

Fig. 7 Statistics (MAE_-dashed lines, MME- solid lines) for the RANSAC application to the fifth-order phase PPS: thin lines - without refinement; thick lines_-with refinement)

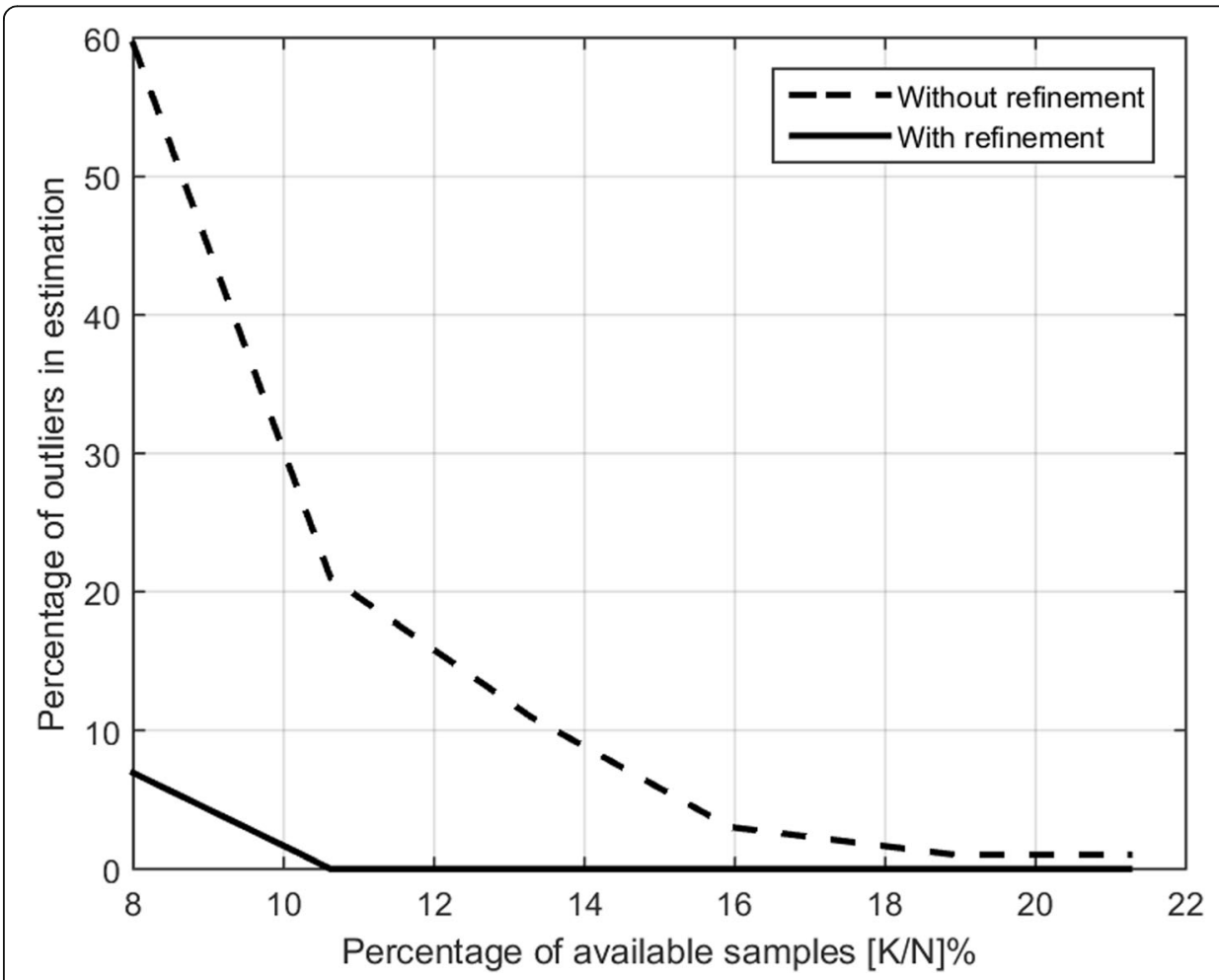

Fig. 8 Percentage of outliers in the IF estimation for the fifth-order PPS: dashed line-without refinement; dashed line-with refinement 
increased all sets to $N_{S}=41$ elements, i.e., search space to $41^{4} \approx 2.83 \times 10^{6}$ elements. It is almost 30 times the number of trials in the RANSAC. The obtained results are summarized in Table 2 with a comparison in terms of the MAE and MME. It can be seen that the direct search strategy with an increase of the search space improves results, but they are not even close enough that the O'Shea refinement can improve accuracy. As we already explained it is required search space of at least $N_{S}=100$ elements for each parameter (three orders of magnitude of the RANSAC number of trials) that the ML, matching pursuit, and other similar strategies produce acceptable results in a majority of problems.

Example 3:

This study is related to a noisy environment. It is a rare case that signals are not subject to some amount of noise, so we have tested the proposed technique for the cubic-phase signal for various amounts of the Gaussian noise. We have performed 100 trials with $K / N=12 \%$ for different variances of the Gaussian noise, Fig. 9. It can be seen that for high noise with $\mathrm{SNR}=-2 \mathrm{~dB}$ (Fig. 9, top row) both techniques with and without refinement achieve a significant number of outliers. For $S N R=2 d B$, the number of outliers in the case of the application of the refinement is small, while for $S N R=6 \mathrm{~dB}$ and $\mathrm{SNR}=10 \mathrm{~dB}$, there are no outliers in the case of the refinement procedure application. Statistical study for 1000 trials is given in Fig. 10. It can be seen that for $S N R \approx 1 d B$, there is a significant drop in the percentage of outliers in the case of the proposed technique with refinement with excellent results in the IF estimation proving that the proposed technique keeps accuracy even in the case of the additive Gaussian noise environment. The obtained results could be further improved by filtering in the O'Shea refinement.

\section{Multicomponent signals}

The proposed technique can be generalized for multicomponent signals with a known parametric components model. The simplest technique is to estimate parameters component-by-component. The strongest component can be estimated firstly and peeled-off from the mixture followed by estimation of subsequent components' parameters. Consider a multicomponent signal with $Q$ FM components:

$$
x(n)=\sum_{q=1}^{Q} x_{q}(n)=\sum_{q=1}^{Q} A_{q} \exp \left[j\left(a_{q, 0}+\sum_{m=1}^{M} a_{q, m} \frac{n^{m}}{m}\right)\right] .
$$

The goal is to estimate signal parameters of all components for an undersampled signal. The procedure is relatively simple: we are estimating the strongest component firstly by the previously described procedure and after estimation of its parameters the

Table 2 MAE and MME for the RANSAC without and with refinement, direct search technique over spaces of $21^{4}$ and $41^{4}$ elements

\begin{tabular}{lllll}
\hline & MAE & MME & MAE[dB] & MME[dB] \\
\hline RANSAC & 0.185 & 1.254 & -7.22 & 0.99 \\
RANSAC+refinement & $1.64 \times 10^{-16}$ & $1.29^{-15}$ & -157.85 & -148.89 \\
Direct search $N_{S}=21$ & 1.831 & 12.739 & 2.63 & 11.05 \\
Direct search $N_{S}=41$ & 1.157 & 1.550 & 0.63 & 1.90 \\
\hline
\end{tabular}




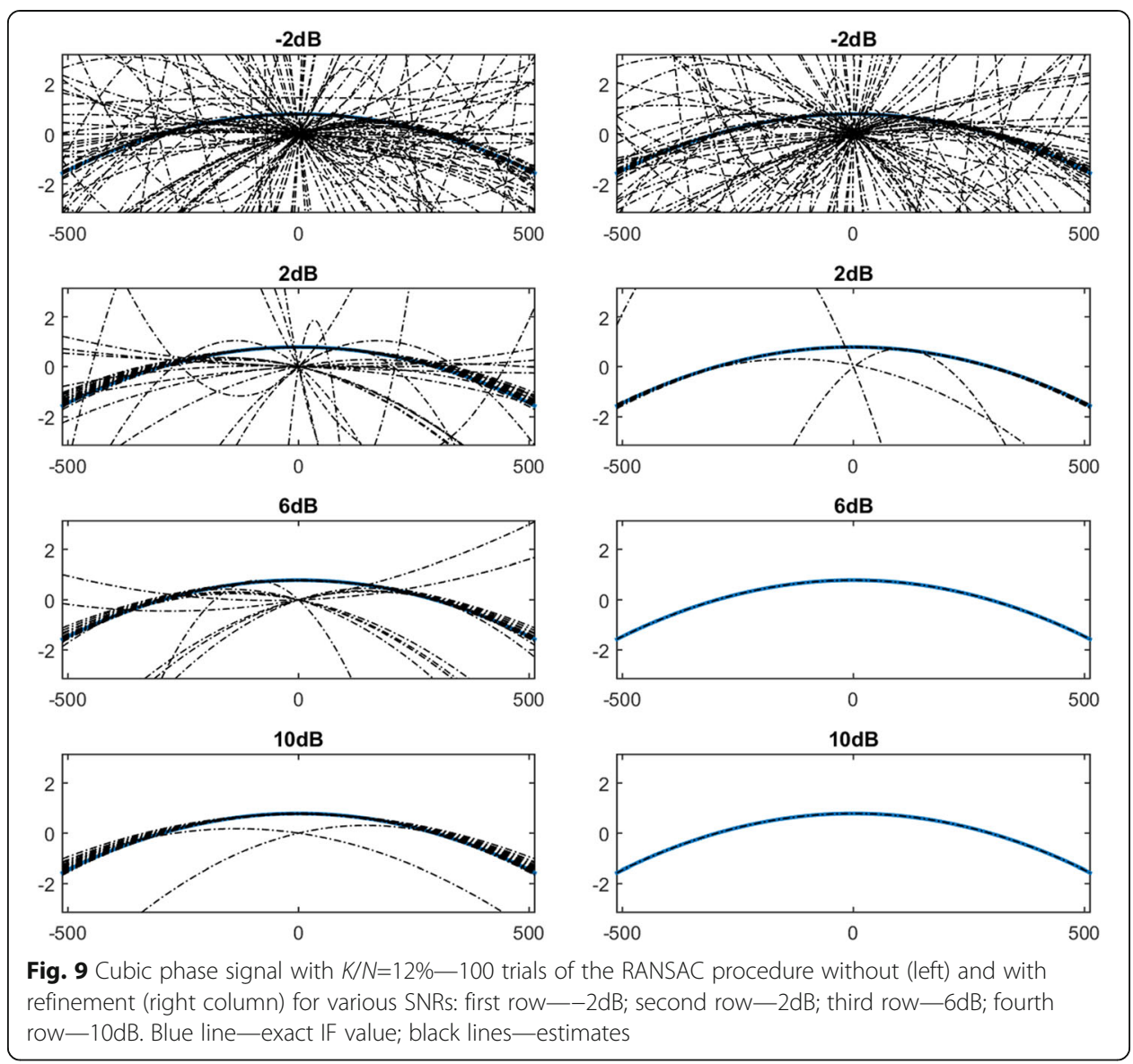

component is removed from the mixture and estimation is performed for weaker components.

Initialization: $y(n)=x(n)$.

For $q=1: Q$

$$
\left[\hat{a}_{q, 1}, \hat{a}_{q, 2}, \ldots, \hat{a}_{q, M}\right]=\mathrm{QML}-\operatorname{RANSAC}(y(n)),
$$

where QML-RANSAC is the procedure previously described in Section III.1.

The $q$ th component is peeled-off from the mixture, firstly by dechirping:

$$
Y(\omega)=\mathrm{FT}\left\{y(n) \exp \left[-j \sum_{m=1}^{M} \hat{a}_{q, m} \frac{n^{m}}{m}\right]\right\} .
$$

followed by setting $Y(0)=0$ (in our experiments, the sample in the origin is removed but a narrow region around can also be neglected) and calculating signal for the next iteration:

$$
y(n)=\operatorname{IFT}\{Y(\omega)\} \exp \left[j \sum_{m=1}^{M} \hat{a}_{q, m} \frac{n^{m}}{m}\right]
$$

where IFT is the inverse FT operator. 


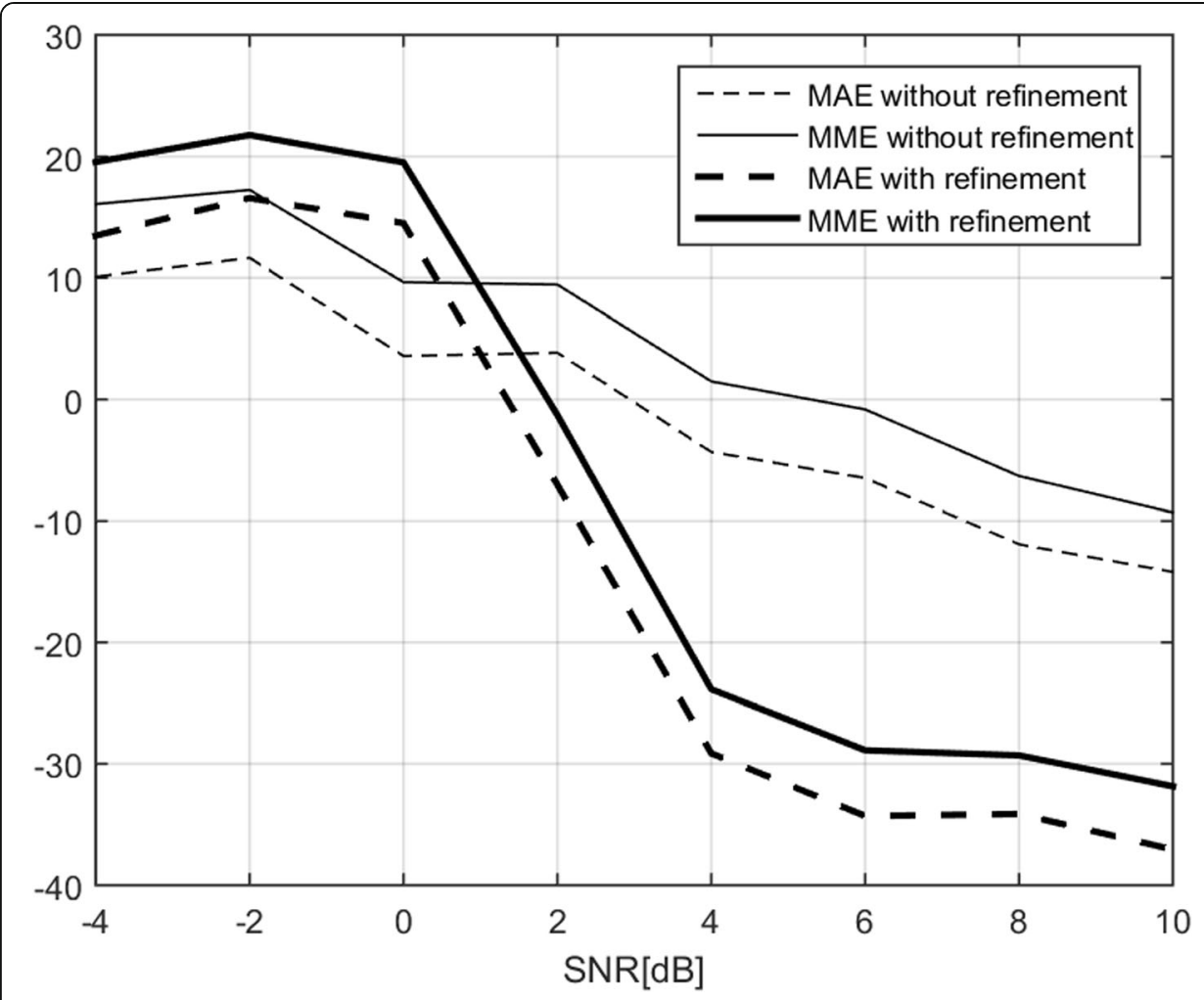

Fig. 10 Statistics (MAE — dashed lines, MME—-solid lines; thin lines—-without refinement; thick line — with refinement) for the RANSAC application to the noisy cubic phase signal with $K / N=12 \%$ in the Gaussian noise environment vs. the SNR.

The technique proposed in [34] can be utilized for improving accuracy in the multicomponent signal parameters' estimation. It is not used in our experiments.

Before presenting some of the simulation results, we have to discuss the possibility to conduct this procedure accurately. As we have already claimed, it can work for signals of different amplitudes where the STFT maximization can be used to estimate parameters of the strongest component. The proposed technique can be also applied for the estimation of separable components in the TF plane. It is possible to estimate intersecting components in the TF plane with the same amplitude but with more trials in the RANSAC procedure than in the case of monocomponent signals. Assume that the percentage of outliers in the IF estimation is $e$. In the worst-case scenario, the remaining 1-e percentage of correct estimates is uniformly distributed on $Q$ signal components (1-e)/Q. The RANSAC requires that all $M$ samples belong to the same component number. The required number of trials should satisfy:

$$
\begin{aligned}
& Q\left(1-((1-e) / Q)^{M}\right)^{\Lambda}=1-p \\
& \Lambda=\log ((1-p) / Q) / \log \left(\left(1-((1-e) / Q)^{M}\right) . .\right.
\end{aligned}
$$

For example, for $p=0.99, e=0.5$, and $Q=2$, it is required $\Lambda=5423$ trials (only 146 for $Q=1$ ) and more than 44000 for $Q=3$. Conditions significantly vary with changes in $M$, $Q, p$, and $e$. 


\section{Example 4.}

We are considering four various mixtures for $Q=2$-component cubic phase signals $(M=3)$ : components separated in TF plane with different amplitudes $A_{1}=1, A_{2}=0.5$; intersecting components and different amplitudes; components of the same amplitudes $A_{1}=A_{2}=1$ but well separated in the TF plane; components with the same amplitude intersecting in the TF plane. Figure 11 demonstrates the STFTs with a window length of 160 samples and IF estimates obtained in the proposed algorithm for $K / N=25 \%$ of available samples for previously described cases. For components of different magnitudes (Fig. 11a-d), firstly the strongest component is estimated and peeled-off following the same procedure on the weaker one. For separable components of the same magnitude (Fig. 11e, f), the first component is estimated by maximizing STFT for $\omega>0$. In the final case of intersecting components of the same magnitude, we have performed IF estimation looking for the STFT maxima position and estimating parameters of the first component. In this case, we do not know which component will be selected in the first QML-RANSAC run but as it can be seen the IF estimation is accurate (Fig. $11 \mathrm{~g}, \mathrm{~h}$ ). In all four cases, it is impossible to visually distinguish between estimates and true IF values. The same holds for $K / N=12 \%$ of available samples with results given in Fig. 12. It can be observed significant deterioration in the STFT representations, but anyway, the IF estimation results are excellent. Finally, for $K / N=6 \%$ of available samples, outliers in the IF estimation can be observed (Fig. 13).

\section{Conclusion}

The QML-RANSAC technique is applied to the parameter estimation of undersampled FM signals. The proposed technique overcomes the main problem in the matching pursuit and greedy strategies generalization to signals with more parameters by employing the RANSAC algorithm. The RANSAC has the advantage of significantly reduced search space comparing direct search techniques. It is shown that without employing

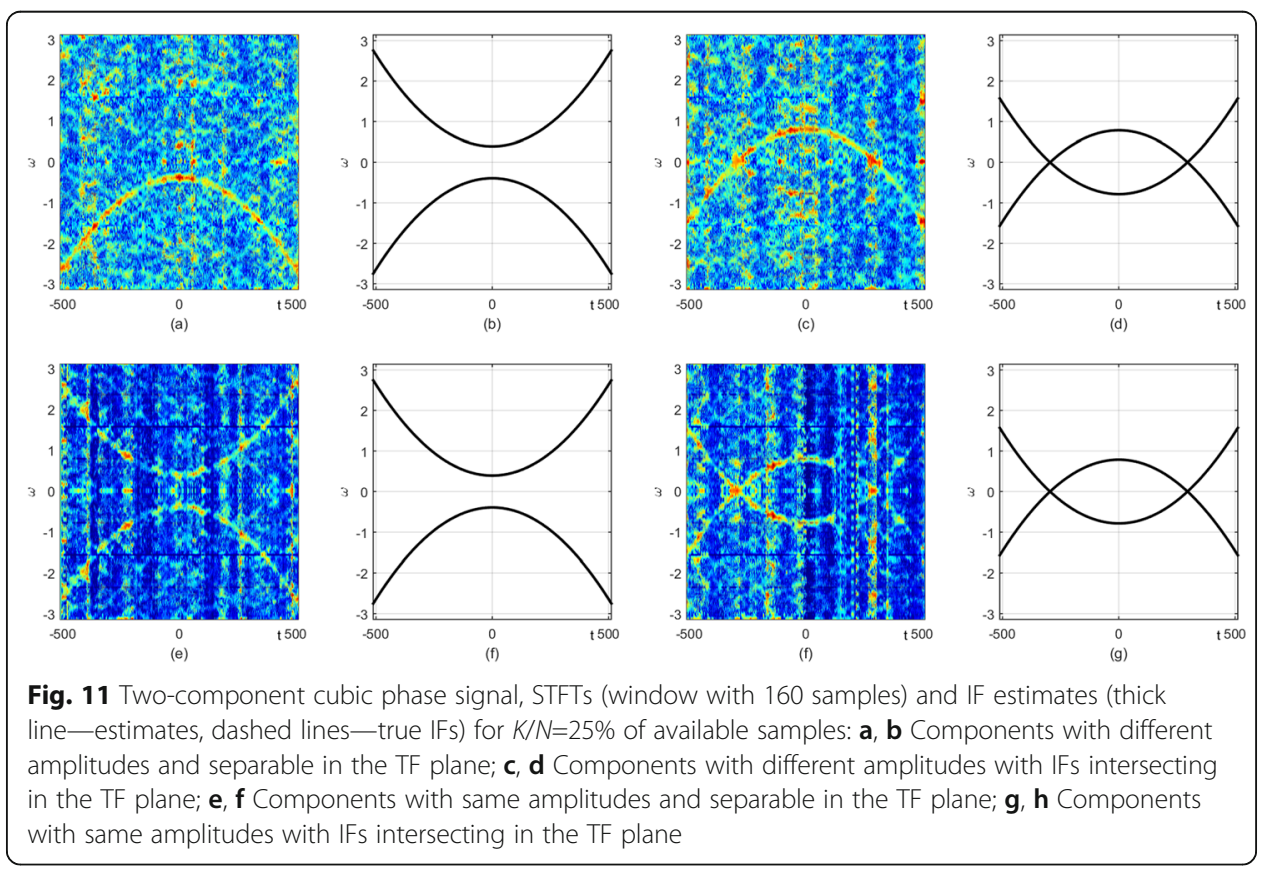




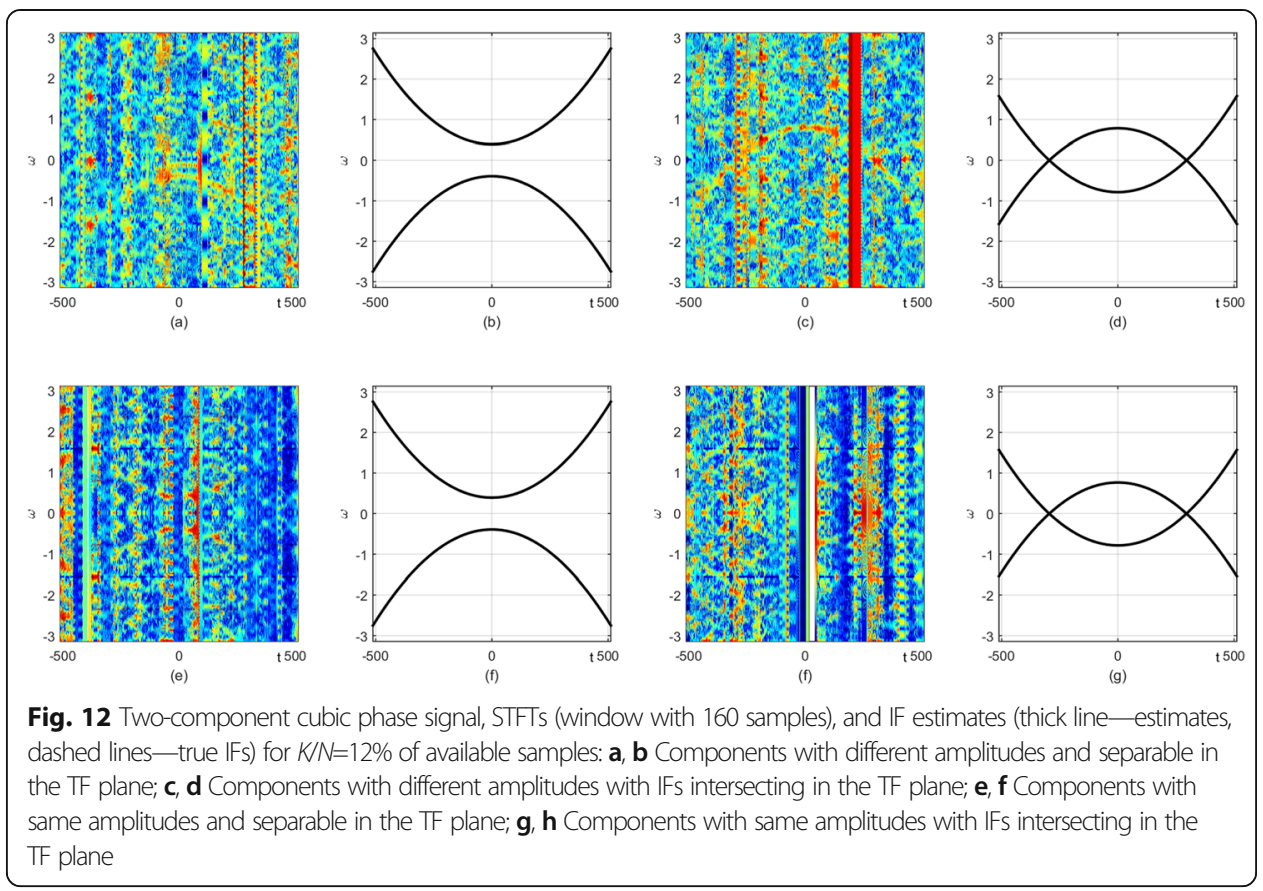

refinement of the phase coefficients mediocre results are obtained while with the O'Shea refinement results are significantly improved reducing the number of outliers in the final estimation for several orders of magnitude. There are potential directions for further development of this technique. Firstly, the O'Shea refinement procedure is developed for signals sampled according to the Nyquist criterion, but here, it is demonstrated that it behaves satisfactorily even for signals with missing samples. It can be expected that the phase estimates interpolation can improve the phase unwrapping procedure. Also, there is room for the employment of downsampled data filtering in

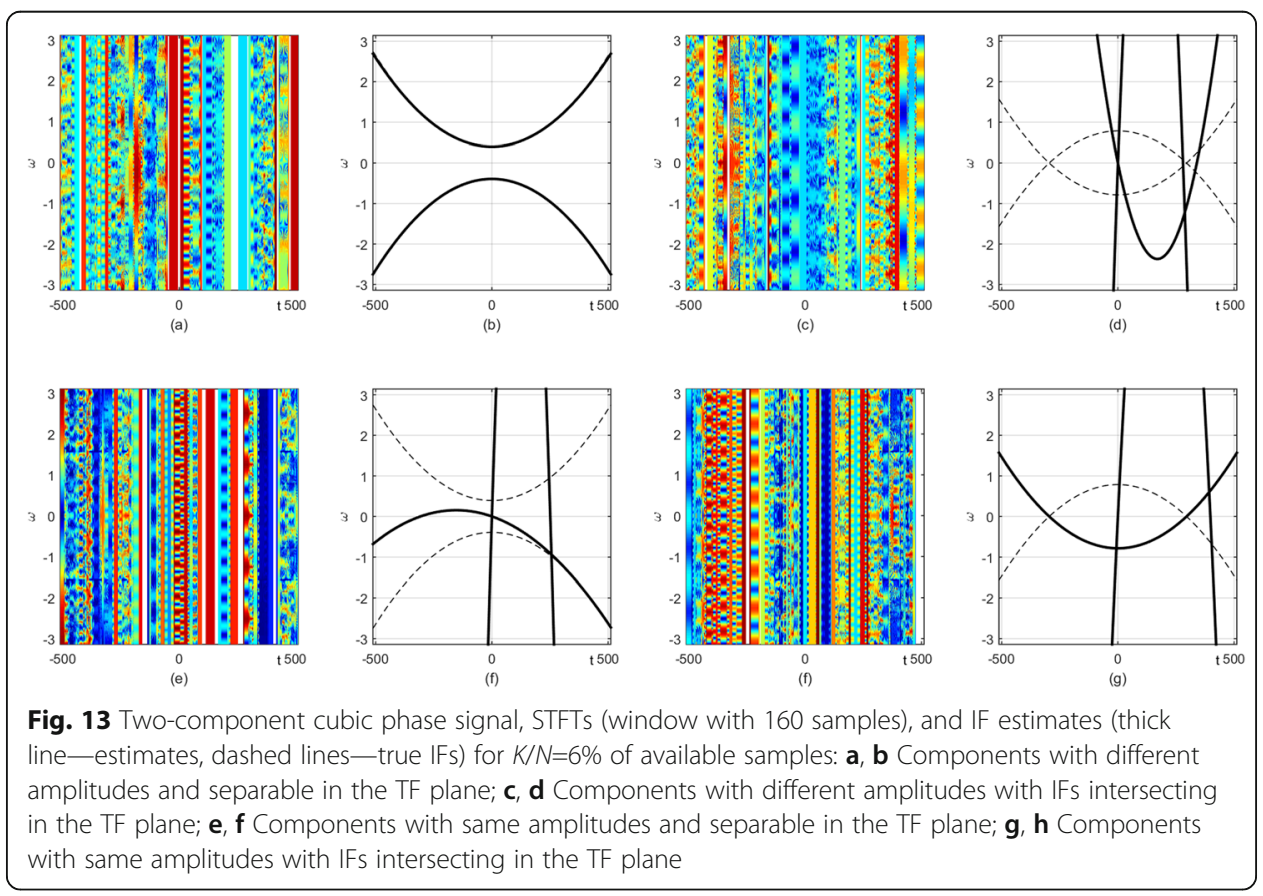


the refinement procedure that is avoided in the present algorithm. Probably, some sort of weighted filtering with weights determined based on the distance to available samples can be helpful. Both the QML and RANSAC are generalized to work for multicomponent signals. Improvement in the multicomponent undersampled signals estimation and reconstruction will be also considered in our future research.

\begin{abstract}
Abbreviations
FM: Frequency modulation; CS: Compressive sensing; RANSAC: Random samples consensus; IF: Instantaneous frequency; TF: Time-frequency; PPS: Polynomial phase signal; QML: Quasi maximum likelihood; ML: Maximum likelihood; STFT: Short-time Fourier transform; SNR: Signal-to-noise ratio; IFT: Inverse Fourier transform; MAE: Mean absolute error; MME: Mean maximal absolute error
\end{abstract}

\title{
Acknowledgements
}

Not applicable

Author's contributions

The author read and approved the final manuscript.

\section{Funding}

There is no research funding supporting this research.

\section{Availability of data and materials}

All experiments are described in detail within a reproducible signal processing framework. Programs are available on request.

\section{Declarations}

\section{Consent for publication}

Not applicable.

\section{Competing interests}

The authors declare that they have no competing interests.

Received: 30 October 2019 Accepted: 6 April 2021

Published online: 17 May 2021

\section{References}

1. R.G. Baraniuk, Compressive sensing. IEEE Signal Process. Mag. 24(4), 118-121 (2007)

2. D.L. Donoho, Compressed sensing. IEEE Trans. Inf. Theory 52(4), 1289-1306 (2006)

3. E. Sejdić, I. Djurović, J. Jiang, Time-frequency feature representation using energy concentration: An overview of recent advances. Dig. Sign. Proc. 19(1), 153-183 (2009)

4. P. Flandrin, P. Borgnat, Time-frequency energy distributions meet compressed sensing. IEEE Trans. Signal Process. 58(6), 2974-2982 (2010)

5. E. Sjedić, I. Orović, S. Stanković, Compressive sensing meets time-frequency: An overview of recent advances in timefrequency processing of sparse signals. Dig. Sign. Proc. 77, 22-35 (2018) https://www.sciencedirect.com/science/article/ pii/S1051200417301665

6. B. Jokanović, M. Amin, Reduced interference sparse time-frequency distributions for compressed observations. IEEE Trans. Signal Process. 63(24), 6698-6709 (2015)

7. B. Boashash, G. Azemi, J.M. O'Toole, Time-frequency processing of nonstationary signals: Advanced TFD design to aid diagnosis with highlights from medical applications. IEEE Signal Process. Mag. 30(6), 108-119 (2013)

8. M.G. Amin, B. Jokanović, Y.D. Zhang, F. Ahmad, A sparsity-perspective to quadratic time-frequency distributions. Dig. Sign. Proc. 46, 175-190 (2015)

9. Y. Wang, J. Xiang, Q. Mo, S. He, Compressed sparse time-frequency feature representation via compressive sensing and its applications in fault diagnosis. Measurement 68, 70-81 (2015)

10. S. Stanković, I. Orović, L.J. Stanković, Polynomial Fourier domain as a domain of signal sparsity. Signal Process. 130, 243$253(2017)$

11. I. Djurović, M. Simeunović, Review of the quasi maximum likelihood estimator for polynomial phase signals. Dig. Sign. Proc. 72, 59-74 (2018)

12. S. Stanković, I. Orović, M. Amin, L-statistics based modification of reconstruction algorithms for compressive sensing in the presence of impulse noise. Signal Process. 93(11), 2927-2931 (2013)

13. I. Orović, S. Stanković, T. Thayaparan, Time-frequency based instantaneous frequency estimation of sparse signals from an incomplete set of samples. IET Sign. Proc. 8(3), 239-245 (2014)

14. E. Sejdić, L.F. Chaparro, in Proc. of NEBEC. Recovering heart sounds from sparse samples (2012), pp. 107-108

15. J. Oh, S. Senay, L.F. Chaparro, Signal reconstruction from nonuniformly spaced samples using evolutionary Slepian transform-based POCS. EURASIP J. Adv. Sign. Proc. 2010, 367317 (2010)

16. S.G. Mallat, Z. Zhang, Matching pursuits with time-frequency dictionaries. IEEE Trans. Signal Process. 41(12), 3397-3415 (1993)

17. L. Stanković, S. Stanković, M. Amin, Missing samples analysis in signals for applications to l-estimation and compressive sensing. Signal Process. 94, 401-408 (2014) 
18. S. Senay, L.F. Chaparro, L. Durak, Reconstruction of nonuniformly sampled time-limited signals using prolate spheroidal wave functions. Signal Process. 89(12), 2585-2595 (2009)

19. D. Angelosante, G.B. Giannakis, N.D. Sidiropoulos, Sparse parametric models for robust nonstationary signal analysis: Leveraging the power of sparse regression. IEEE Signal Process. Mag. 30(6), 64-73 (2013)

20. I. Djurović, A WD-RANSAC instantaneous frequency estimator. IEEE Sign. Proc. Lett. 23(5), 757-761 (2016)

21. I. Djurović, QML-RANSAC: PPS and FM signals estimation in heavy noise environments. Sign. Proc. 130, 142-151 (2017)

22. I. Djurović, QML-RANSAC IF estimator for overlapping multicomponent signals in the TF plane. IEEE Sign. Proc. Lett. 25(3), 447-451 (2018)

23. I. Djurović, L.J. Stanković, J.F. Böhme, Robust L-estimation based forms of signal transforms and time-frequency representations. IEEE Trans. Sign. Proc. 51(7), 1753-1761 (2003)

24. L.J. Stanković, I. Djurović, S. Stanković, M. Simeunović, M. Daković, Instantaneous frequency in time-frequency analysis: Enhanced concepts and performance of estimation algorithms. Dig. Sign. Proc. 35, 1-13 (2014)

25. P. O'Shea, On refining polynomial phase signal parameter estimates. IEEE Trans. Aerosp. Electron. Syst. 46(3), 978-987 (2010)

26. I. Djurović, L.J. Stanković, Quasi maximum likelihood estimator of polynomial phase signals. IET Sign. Proc. 13(4), 347-359 (2014)

27. I. Djurović, Quasi ML algorithm for 2-D PPS estimation. Multidimens. Sign. Syst. 28(2), 371-389 (2017)

28. N.R. Brnović, I. Djurović, V.N. Ivanović, M. Simeunović, Hardware implementation of the quasi maximum likelihood estimator core for polynomial phase signals. IET Circuits Devices Syst. 13(2), 131-138 (2019)

29. V. Katkovnik, I. Djurović, L.J. Stanković, in Time-Frequency Signal Analysis and Applications, 2nd edn., ed. by B. Boashash ISBN: 9780123984999. Robust Time-Frequency Distributions (Academic, 2015), pp. 539-546

30. A. Roenko, V. Lukin, I. Djurović, An overview of the adaptive robust DFT. Eurasip J. Adv. Sign. Proc.special issue "Robust Processing of Nonstationary Signals", 595071 (2010) https://asp-eurasipjournals.springeropen.com/articles/10.1155/2010/ 595071

31. I. Djurović, On parameters of the QML PPS estimator. Signal Process. 116, 1-6 (2015)

32. I. Djurović, M. Simeunović, B. Lutovac, Are genetic algorithms useful for the parameter estimation of FM signals. Dig. Sign. Proc. 22(6), 1137-1144 (2012)

33. R. Beneš, M. Hasmanda, K. Říha, Non-linear RANSAC method and its utilization. ElectroRevue 2(4), 7-14 (2011)

34. D.S. Pham, A.M. Zoubir, Analysis of multicomponent polynomial phase signals. IEEE Trans. Sign. Proc. 55(1), 56-65 (2007)

\section{Publisher's Note}

Springer Nature remains neutral with regard to jurisdictional claims in published maps and institutional affiliations.

\section{Submit your manuscript to a SpringerOpen ${ }^{\circ}$ journal and benefit from:}

- Convenient online submission

- Rigorous peer review

- Open access: articles freely available online

High visibility within the field

- Retaining the copyright to your article

Submit your next manuscript at $\boldsymbol{\nabla}$ springeropen.com 